ISSN 0103-8478

\title{
Características endócrinas, metabólicas e indicadoras da qualidade da carne em bovinos Nelore castrados e não castrados
}

\author{
Neuroendocrine, metabolic and meat quality traits in castrated and non-castrated Nellore cattle
}

\author{
Bárbara Silva ${ }^{{ }^{*}}$ Mirele Daiana Poleti ${ }^{\mathrm{I}}$ \\ Cristina Tschorny Moncau ${ }^{\mathrm{I}}$ Alessandra Fernandes Rosa ${ }^{\mathrm{I}}$ \\ Saulo da Luz e Silva ${ }^{I}$ Júlio César de Carvalho Balieiro ${ }^{\mathrm{I}}$
}

RESUMO

O objetivo deste estudo foi avaliar características endócrinas (ACTH e cortisol plasmático), metabólicas (glicogênio e lactato muscular) e indicadoras de qualidade da carne em bovinos Nelore castrados e não castrados. Foram abatidos 130 animais ( 78 castrados e 52 não castrados), entre os anos de 2009 e 2011. Amostras de sangue foram coletadas, in vivo e post mortem (IV e PM), para mensurações endócrinas. Para os metabólitos, foram coletadas amostras do músculo Longissimus dorsi (LD). Para as análises fisico-químicas, três bifes do músculo $L D$ (entre a $10^{a}$ e $12^{a}$ costelas) foram maturados por um, sete e 14 dias. $O$ pH e a temperatura dos animais foram mensurados a uma e 24 horas PM. As análises estatísticas foram realizadas por meio do procedimento de modelos mistos, com auxilio do programa Statistical Analysis System. Observou-se que as concentrações de cortisol (IV e PM) e lactato muscular foram superiores $(P<0,05)$ nos animais castrados em relação aos não castrados. Animais castrados apresentam menores valores de pH 24 horas e carnes mais macias $(P<0,05)$ em todos os tempos de maturação, quando comparados aos não castrados.

Palavras-chave: $A C T H$, bovinos de corte, cortisol, glicogênio, lactato.

\section{ABSTRACT}

The aim of this study was to evaluate neuroendocrine (plasma ACTH and cortisol), metabolic (muscle glycogen and lactate) and meat quality characteristics in castrated and noncastrated Nellore cattle. To this end, 130 animals were slaughtered (78 castrated and 52 non-castrated), between the years 2009 and 2011. Blood samples were collected, in vivo and post mortem (IV and PM), for endocrine measurements. For the metabolites, samples were collected from Longissimus dorsi (LD) muscle. For physical-chemical analysis, three steaks from $L D$ muscle (between $10^{\text {th }}$ and $12^{\text {th }}$ rib) were aged by one, seven and 14 days. The $\mathrm{pH}$ and temperature of the animals were measured at one and 24 hours PM. Statistical analyzes were performed using mixed models of the Statistical Analysis System software. It was observed that muscle cortisol (IV and PM) and lactate concentrations were higher $(P<0.05)$ in castrated compared to non-castrated animals. Castrated had lower $\mathrm{pH} 24$ hours values and tender meat $(P<0.05)$ in all times aging compared to non-castrated animals.

Key words: $A C T H$, beef cattle, cortisol, glycogen, lactate.

\section{INTRODUÇÃO}

A preocupação na definição e quantificação do estresse, quando relacionado com a produção e o bem-estar animal, tem recebido atenção especial nos últimos anos. A resposta endócrina ao estresse é decorrente da ativação do eixo hipotálamo-pituitáriaadrenal (HPA). O eixo HPA controla a síntese e a liberação de glicocorticoides, mineralocorticoides e hormônios sexuais. Em situações estressantes, o eixo HPA estimula o aumento da secreção de ACTH, elevando as concentrações de cortisol circulante (JURUENA et al., 2004).

O manejo pré-abate tem sido apontado como importante responsável pela melhoria da qualidade da carne. Condições inadequadas de manejo pré-abate podem comprometer o metabolismo post mortem. No momento do abate, devido à interrupção do suprimento de oxigênio no músculo, é iniciado o processo de glicólise anaeróbica. O processo consiste na quebra do glicogênio armazenado no tecido em glicose, estimulando a formação de lactato e consequente queda do pH (KOÉWIN-PODSIADLA et al., 2006).

\footnotetext{
IFaculdade de Zootecnia e Engenharia de Alimentos (FZEA), Universidade de São Paulo (USP), 13635-900, Pirassununga, SP, Brasil. E-mail: silva.barbara@zootecnista.com.br.*Autor para correspondência. 
Em bovinos, a glicólise se desenvolve lentamente, com $\mathrm{pH}$ inicial em torno de 7,0, caindo para 6,4-6,8 as 5 horas e para 5,5-5,8 as 24 horas post mortem (WULF et al., 2011). Entretanto, se ocorrer deficiência nas reservas de glicogênio muscular, ocorrerá uma menor formação de lactato e o $\mathrm{pH}$ final permanecerá alto, fazendo com que todo o processo de transformação de músculo em carne seja prejudicado. Segundo WULF et al. (2011), o estresse pré-abate dos animais é o maior causador da diminuição do glicogênio muscular, acarretando em carnes classificadas como DFD (Dark, Firm and Dry).

Outro fator que influencia a qualidade da carne dos animais é a condição sexual. Animais não castrados apresentam carcaça mais magra, carne mais escura, mais dura e de pior palatabilidade que os castrados (FIELD, 1971).

Vários trabalhos foram realizados visando encontrar indicadores para caracterização do nível de estresse em animais. Em revisão, GRANDIN (1997) citou o cortisol como indicador de estresse agudo. MÖSTL \& PALME (2002) e KANITZ et al. (2009) também trabalharam com concentrações de cortisol para determinação do nível de estresse dos animais.

Com respeito à associação do estresse com a qualidade da carne em diferentes condições sexuais, MORGAN et al. (1993), trabalhando com bovinos em diferentes condições sexuais, verificaram que a carne dos animais não castrados apresentou valores de força de cisalhamento de $4,9 \mathrm{~kg}$, superior aos $4,2 \mathrm{~kg}$ dos animais castrados. RODRIGUES \& ANDRADE (2004) também encontraram carne mais macia nos animais castrados em relação aos não castrados.

Pelo exposto, objetivou-se avaliar características endócrinas, metabólicas e indicadoras da qualidade da carne de bovinos Nelore castrados e não castrados.

\section{MATERIAL E MÉTODOS}

Este trabalho foi desenvolvido de acordo com as normas estabelecidas no Regimento Geral do Comitê de Ética em Experimentação Animal da Faculdade de Zootecnia e Engenharia de Alimentos da Universidade de São Paulo.

Cento e trinta bovinos da raça Nelore, 78 machos castrados e 52 machos não castrados, foram recriados a pasto e terminados em confinamento $(59,79$, 114 e 128 dias), pelo período de 2009 a 2011. Em cada ano, foram criados animais de diferentes safras, sendo 43 animais em 2009, 43 em 2010 e 44 em 2011. Os animais foram alimentados com dietas de alto concentrado (85\%), composto por milho, farelo de soja, casca de soja, glúten de milho, sal mineral e ureia, contendo $14,6 \%$ de proteína bruta e $74,5 \%$ de NDT, e bagaço de cana de açúcar (15\%) como fonte de volumoso.

Ao final de cada período de confinamento, os animais foram transportados para um abatedouro a 200 metros de distância, atordoados com pistola pneumática e abatidos por meio da incisão da veia jugular e artéria carótida para sangria. Os animais foram abatidos com idade de aproximadamente 24 meses e peso vivo final médio de $508 \mathrm{~kg}$. Após o abate, as carcaças foram armazenadas a $4^{\circ} \mathrm{C}$ por 24 horas para posterior desossa.

Amostras de sangue dos animais para análises endócrinas foram coletadas em tubos heparinizados em dois períodos, in vivo e post mortem (IV e PM), sendo o primeiro na pesagem dos animais no início do período de confinamento por meio de punção na veia jugular e o segundo durante a exsanguinação no abate. Os hormônios adrenocorticotrófico (ACTH) e cortisol foram analisados no plasma por meio de kits imunoenzimáticos da Biomerica e Monobind, conforme as instruções dos fabricantes.

Aproximadamente 30 minutos post mortem, uma amostra do músculo Longissimus dorsi (LD) foi retirada e imediatamente armazenada em Nitrogênio líquido, para mensuração dos metabólitos. As amostras foram desproteinizadas de acordo com a metodologia descrita por RAMOS \& GOMIDE (2007). O lactato e o glicogênio foram determinados por espectofotometria com os kits de Análise de Lactato e Glicogênio Enzychrom (BioAssay Systems, Hayward, USA), conforme recomendações do fabricante.

$\mathrm{O} \mathrm{pH}$ e a temperatura foram determinados a uma e 24 horas post mortem no músculo Longissimus dorsi, com o auxílio de um pHmetro portátil (NT-PHP; Tecnal). No momento da desossa, amostras do mesmo músculo foram retiradas (três bifes de $2,5 \mathrm{~cm}$ de largura), empacotadas a vácuo e mantidas refrigeradas em câmara fria a $2^{\circ} \mathrm{C}$ para maturar por períodos de um, 
sete e 14 dias. Subsequentemente, cor, força de cisalhamento (FC) e perdas por cozimento (PPC) nos três tempos de maturação foram determinados. A FC (kg) foi mensurada utilizando-se o aparelho Warner Bratzler Shear Force, como a média de 6 cilindros de cada bife, segundo o protocolo AMSA (1995). As PPC foram determinadas pela diferença de peso da amostra antes $\left(\mathrm{P}_{\mathrm{i}}\right)$ e depois $\left(\mathrm{P}_{\mathrm{f}}\right)$ do cozimento, ou seja, $\left[\mathrm{PPC}=\left(\mathrm{P}_{\mathrm{i}}-\mathrm{P}_{\mathrm{f}}\right) / \mathrm{P}_{\mathrm{i}}\right]$ expressa em \%. A cor objetiva $\left(L^{*}, a^{*}, b^{*}\right)$ foi determinada com o auxílio de um colorímetro portátil (CM-2500d, Konica Minolta).

As análises estatísticas foram realizadas com auxílio do procedimento PROC MIXED do programa Statistical Analysis System, versão 9.1.3 (SAS Institute Inc., Cary, NC, USA), de acordo com o seguinte modelo misto:

$$
Y_{i j k l}=\mu+R_{i}+S A_{j}+C_{k}+e_{i j k l}
$$

Em que, $Y_{i j k l}=$ e o valor para determinada variável endócrina ou relacionada ao potencial glicolítico ou variável indicadora da qualidade da carne observada no animal $l$, de condição sexual $k$, de ano-período de abate $j$ e filho do reprodutor $i ; \mu=$ constante comum para todas as observações (média); $R_{i}=$ efeito aleatório do reprodutor $i$, com média 0 e variância $\sigma_{\mathrm{R}}^{2}$, sendo $i=1,2, \ldots, 28 ; S A_{j}=$ efeito fixo do ano e período de abate $j$, sendo $j=1$ (20091), 2(2009-2), 3(2009-3), 4(2009-4), 5(2010-1), ..., 12(2011-4); $C_{k}=$ efeito fixo da condição sexual $k$ do animal, sendo $k=1$ (não castrado) ou 2(castrado); e, $e_{i j k l}=$ é o erro aleatório associado à variável endócrina ou relacionada ao potencial glicolítico ou variável indicadora da qualidade da carne observada no tipo de carne $l$, do animal de condição sexual $k$, no ano e ordem de abate $j$ e filho do reprodutor $i$, suposto normal e independentemente distribuído, com média 0 e variância $\sigma_{e}^{2}$.

Para o modelo acima descrito, quando observado efeito significativo para a fonte de variação condição sexual, o Teste F da ANOVA foi considerado discriminatório.

Para avaliar as possíveis relações existentes entre as variáveis endócrinas, metabólicas e indicadoras da qualidade da carne, foram realizadas análises de correlação Momento-Produto de Pearson, dentro de cada condição sexual avaliada.

\section{RESULTADOS E DISCUSSÃO}

Os valores de cortisol IV e PM para bovinos Nelore castrados foram maiores $(\mathrm{P}<0,0001)$ em relação a não castrados, assim como os valores de lactato muscular $(\mathrm{P}<0,05)$ e cor de carne $\left(\mathrm{a}^{*} \mathrm{e} \mathrm{b}^{*}\right)$ nos três tempos de maturação $(\mathrm{P}<0,05)$ (Tabela 1).

A condição sexual influenciou a concentração de lactato muscular $(\mathrm{P}<0,05)$. Segundo RAMOS \& GOMIDE (2007), a concentração de ácido lático média no músculo LD logo após o abate deve ser em torno de 6 a $16 \mu \mathrm{mol} \mathrm{g}^{-1}$. Os animais castrados tiveram maior conteúdo de lactato $\left(16,90 \pm 0,707 \mu \mathrm{mol} \mathrm{g}^{-1}\right)$ em relação aos não castrados $\left(14,35 \pm 0,853 \mu \mathrm{mol} \mathrm{g}^{-1}\right)$. $\mathrm{O}$ acúmulo do lactato no músculo é decorrente da interrupção da circulação sanguínea que remove os produtos metabólicos finais da atividade muscular. $\mathrm{O}$ ácido láctico acumula no tecido e consequentemente diminui o pH (PAREDI et al., 2012).

Os valores determinados para lactato podem explicar as diferenças significativas encontradas para $\mathrm{pH} 24$ horas entre as condições sexuais $(\mathrm{P}>0,01)$. Os maiores valores de lactato para castrados refletiram em um menor $\mathrm{pH} 24$ horas nesses animais. A média dos valores de $\mathrm{pH} 24$ horas post mortem para ambas as condições sexuais estava dentro da faixa normal para carne bovina $(5,4-5,8)$ (LAWRIE, 2005).

O metabolismo de energia e a queda do pH estão entre as mais importantes alterações que influenciam a qualidade da carne e que afetam seu valor comercial (PAREDI et al., 2012), uma vez que o pH influencia a cor da carne e a capacidade de retenção de água (HUFF-LONERGAN \& LONERGAN, 2005). Carnes mais vermelhas são mais atrativas para o consumidor, pois são associadas com frescor e integridade do produto (MANCINI \& HUNT, 2005).

Em relação à cor da carne, os valores de $a^{*}$ e $b^{*}$ foram significativamente superiores nos animais castrados, enquanto os valores de $\mathrm{L}^{*}$ apresentaram diferenças significativas apenas no primeiro dia de maturação $(\mathrm{P}<0,05)$, semelhante aos achados de RODRIGUES \& ANDRADE (2004). Em concordância com o presente estudo, JEREMIAH et al. (1991), VAZ \& RESTLE (2000) também encontraram que animais castrados têm menor $\mathrm{pH}$ de carcaça fria e carne de coloração mais clara, 
Tabela 1 - Efeito da condição sexual sobre as características endócrinas, metabólicas e de qualidade de carne de bovinos Nelore.

\begin{tabular}{|c|c|c|c|c|c|}
\hline \multirow{2}{*}{ Variável $^{1}$} & \multicolumn{4}{|c|}{ 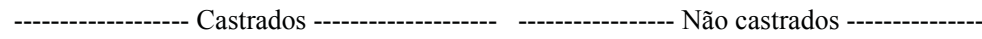 } & \multirow{2}{*}{ Valor de $\mathrm{P}$} \\
\hline & Média & Erro padrão & Média & Erro padrão & \\
\hline Cortisol $I V\left(\mu \mathrm{g} \mathrm{dL}^{-1}\right)$ & $5,28^{\mathrm{A}}$ & 0,269 & $3,67^{\mathrm{B}}$ & 0,315 & $<0,0001$ \\
\hline Cortisol $P M\left(\mu \mathrm{g} \mathrm{dL}^{-1}\right)$ & $7,76^{\mathrm{A}}$ & 0,318 & $5,40^{\mathrm{B}}$ & 0,373 & $<0,0001$ \\
\hline ACTH $I V\left(p g \mathrm{~mL}^{-1}\right)$ & $16,62^{\mathrm{A}}$ & 1,699 & $14,17^{\mathrm{A}}$ & 2,019 & 0,3540 \\
\hline ACTH $P M\left(p g L^{-1}\right)$ & $10,23^{\mathrm{A}}$ & 1,632 & $10,49^{\mathrm{A}}$ & 1,930 & 0,9141 \\
\hline Glicogênio & $17,13^{\mathrm{A}}$ & 0,833 & $15,71^{\mathrm{A}}$ & 1,005 & 0,2775 \\
\hline Lactato & $16,90^{\mathrm{A}}$ & 0,707 & $14,35^{\mathrm{B}}$ & 0,853 & 0,0237 \\
\hline$L * 1 D$ & $33,55^{\mathrm{A}}$ & 0,512 & $32,01^{\mathrm{B}}$ & 0,599 & 0,0320 \\
\hline$a * 1 D$ & $18,94^{\mathrm{A}}$ & 0,321 & $17,97^{\mathrm{B}}$ & 0,375 & 0,0300 \\
\hline$b * 1 D$ & $15,95^{\mathrm{A}}$ & 0,227 & $14,97^{\mathrm{B}}$ & 0,275 & 0,0070 \\
\hline$L * 7 D$ & $36,05^{\mathrm{A}}$ & 0,557 & $35,19^{\mathrm{A}}$ & 0,631 & 0,2118 \\
\hline$a * 7 D$ & $17,15^{\mathrm{A}}$ & 0,294 & $15,67^{\mathrm{B}}$ & 0,348 & 0,0009 \\
\hline$b * 7 D$ & $15,93^{\mathrm{A}}$ & 0,244 & $14,72^{\mathrm{B}}$ & 0,290 & 0,0012 \\
\hline $\mathrm{L} * 14 \mathrm{D}$ & $35,42^{\mathrm{A}}$ & 0,482 & $34,26^{\mathrm{A}}$ & 0,593 & 0,1263 \\
\hline$a * 14 D$ & $18,62^{\mathrm{A}}$ & 0,283 & $17,26^{\mathrm{B}}$ & 0,339 & 0,0012 \\
\hline$b^{*} 14 \mathrm{D}$ & $16,40^{\mathrm{A}}$ & 0,203 & $15,24^{\mathrm{B}}$ & 0,248 & 0,0003 \\
\hline $\mathrm{pH} 1 \mathrm{H}$ & $7,85^{\mathrm{A}}$ & 1,173 & $7,92^{\mathrm{A}}$ & 1,173 & 0,4168 \\
\hline $\mathrm{pH} 24 \mathrm{H}$ & $5,51^{\mathrm{B}}$ & 0,026 & $5,62^{\mathrm{A}}$ & 0,031 & 0,0062 \\
\hline PPC1D & $23,63^{\mathrm{A}}$ & 0,463 & $24,12^{\mathrm{A}}$ & 0,554 & 0,4970 \\
\hline PPC7D & $25,19^{\mathrm{A}}$ & 0,724 & $25,70^{\mathrm{A}}$ & 0,877 & 0,5815 \\
\hline PPC14D & $24,91^{\mathrm{A}}$ & 0,579 & $24,44^{\mathrm{A}}$ & 0,668 & 0,5391 \\
\hline FC1D & $9,08^{\mathrm{A}}$ & 0,195 & $9,62^{\mathrm{A}}$ & 0,236 & 0,0840 \\
\hline FC7D & $7,33^{\mathrm{B}}$ & 0,196 & $8,64^{\mathrm{A}}$ & 0,236 & $<0,0001$ \\
\hline FC14D & $6,11^{\mathrm{B}}$ & 0,215 & $6,81^{\mathrm{A}}$ & 0,246 & 0,0123 \\
\hline
\end{tabular}

${ }^{1}$ Cortisol $I V$ e ACTH $I V=$ concentração desses hormônios determinadas em amostras de sangue coletadas dos animais no confinamento (in vivo); Cortisol $P M$ e ACTH $P M=$ concentração desses hormônios determinadas em amostras de sangue coletadas no momento da sangria no abate (post mortem); Glicogênio e lactato em $\mu \mathrm{molg}^{-1}$ de tecido; $\mathrm{PPC}=$ perdas por cozimento, em $\%$; $\mathrm{FC}=$ valores de força de cisalhamento em quilogramas.

${ }^{*}$ Médias na mesma linha e seguidas por uma mesma letra maiúscula não diferem entre si, ao nível de significância conjunta de 5\%, pelo teste $t$ de Student.

demonstrando que animais castrados tiveram um maior metabolismo energético muscular, gerando maior acúmulo de lactato, menor pH 24 horas e uma cor de carne mais vermelha $\left(a^{*}\right)$ e mais amarela $\left(b^{*}\right)$. Os valores médios de $\mathrm{L}^{*}, \mathrm{a}^{*} \mathrm{e} \mathrm{b}^{*}$ apresentados foram semelhantes aos observados por PINTO et al. (2008).

Segundo COSTA et al. (2002) a condição sexual e a idade estão entre os fatores que explicam a variação na cor da carne. No estudo de MACH et al. (2009), não houve diferenças para pH do músculo Longissimus thoracis entre as condições sexuais, porém animais não castrados tiveram uma carne mais escura $\left(<\mathrm{L}^{*}\right)$, menos vermelha $\left(<\mathrm{a}^{*}\right)$ e menos amarela $\left(<\mathrm{b}^{*}\right)$.

A cor mais escura em carnes de animais não castrados é frequentemente relatada. Entretanto, níveis similares de mioglobina são encontrados em músculos de animais castrados e não castrados (FIELD, 1971). A cor mais escura atribuída a não castrados pode ser atribuída a um $\mathrm{pH}$ mais alto, recorrente de um temperamento mais excitável em relação aos castrados e, portanto, altas taxas de glicólise ante mortem (LAWRIE, 2005).

Não se observou diferença significativa entre as condições sexuais para a característica de perda de água por cozimento para nenhum dos tempos de maturação. Todavia, para a característica de força de cisalhamento, relacionada com maciez da carne, observou-se que animais não castrados apresentaram carne com maior FC em todos os tempos de maturação, e em ambas as condições sexuais a FC diminuiu do primeiro para o 14ํ dia de maturação (Tabela 1). FIELD (1971), em uma revisão sobre castrados e não 
castrados, relatou diversos estudos que demonstram que carne de animais não castrados é menos macia do que de animais castrados.

A menor maciez para animais não castrados pode ser atribuída a diferenças no marmoreio, cobertura de gordura e a quantidade e solubilidade de colágeno (DIKEMAN et al., 1986). Além disso, MORGAN et al. (1993), estudando o efeito da castração sobre o sistema proteolítico da calpaína, identificou que bovinos não castrados têm maior atividade da calpastatina no músculo Longissimus e menor proteólise durante os primeiros sete dias post mortem, podendo ser atribuída a uma diminuição da maciez.

Entretanto, VAZ \& RESTLE (2000), utilizando painel de avaliadores treinados e força de cisalhamento para carne de bovinos Hereford, relataram que animais não castrados têm uma carne mais macia e de melhor palatabilidade que animais castrados.

Em outros estudos, animais não castrados apresentam carcaça mais magra, carne mais escura, mais dura e de pior palatabilidade que os castrados (RESTLE et al., 1994; RESTLE et al., 1996).

Uma maior concentração de cortisol nos animais castrados foi observada em ambos os períodos de coleta (IV e PM), em comparação aos animais não castrados, o que permite-nos inferir que a castração pode estar influenciando de alguma maneira na rota metabólica de síntese desse hormônio, como se a supressão da produção do hormônio sexual, testosterona, estimulasse a produção do hormônio cortisol, como um mecanismo compensatório do organismo.

O cortisol é o principal hormônio do eixo hipotálamo-pituitária-adrenal, o mais importante sistema neuroendócrino de resposta ao estresse e, em termos de produção animal, o efeito metabólico do cortisol favorece a deposição de lipídios no tecido adiposo, em detrimento das proteínas do músculo e outros tecidos. Um aumento no cortisol diminui o peso da carcaça, a taxa de crescimento e a eficiência alimentar. Porém, o cortisol tem também efeitos positivos sobre a habilidade de combinar alta produção com resistência a estressores, ou seja, melhor adaptação do animal (MORMÉDE et al., 2011).

Dessa maneira, é possível observar que um dos fatores que levam a animais castrados terem maior deposição de gordura, menor peso de carcaça, carne de melhor coloração e mais macia, como citados em diversos trabalhos na literatura (DIKEMAN et al., 1986; MORGAN et al., 1993; VAZ \& RESTLE, 2000; MACH et al., 2009), podem ser decorrentes do aumento da concentração do cortisol nesses animais após a castração. A melhor qualidade de carne confirma uma melhor adaptação a uma situação estressora, como o abate, e, portanto, não interferindo na glicólise ante mortem, que afetaria a queda do $\mathrm{pH}$ e consequentemente, demais características como cor, perdas por cozimento e maciez.

De maneira geral, as estimativas dos coeficientes de correlação entre as variáveis endócrinas e metabólicas e as características de qualidade de carne para os animais castrados e não castrados foram de baixa à média magnitude (Tabela 2). $\mathrm{O}$ cortisol e o ACTH IV apresentaram correlações com os valores de $\mathrm{a}^{*}$ aos sete e 14 dias de maturação $(\mathrm{P}<0,01)$ e correlação de $-0,323$ entre cortisol PM e L* aos 14 dias $(\mathrm{P}<0,01)$, nos animais castrados. Nos não castrados, apenas o cortisol apresentou correlação de 0,342 com a* aos 14 dias $(\mathrm{P}<0,05)$.

Além dessas, o ACTH IV exibiu correlações negativas com $\mathrm{L}^{*}$ em todos os dias de maturação $(\mathrm{P}<0,01)$ e positiva com a* no primeiro dia $(\mathrm{r}=0,445, \mathrm{P}<0,0001)$, nos animais castrados.

Em relação aos metabólitos, o lactato apresentou correlação positiva com L* em todos os tempos de maturação $(\mathrm{P}<0,05)$ para as duas condições sexuais, além de correlações negativas com a* no primeiro e $14^{\circ}$ dia e $b^{*}$ no $7^{\circ}$ dia em machos não castrados $(\mathrm{P}<0,05)$. $\mathrm{O}$ glicogênio foi correlacionado com b* em todos os tempos de maturação e FC aos 7 dias em animais não castrados $(\mathrm{P}<0,05)$.

Diante do exposto, é possível averiguar que os achados que animais castrados têm uma melhor qualidade de carne em relação à cor e maciez, comparado a animais não castrados, podem ser decorrentes da atividade hormonal, bem como a influência destes sobre o metabolismo glicolítico muscular post mortem. Este, por sua vez, gera lactato que promove a queda do $\mathrm{pH}$ da carcaça e, consequentemente, influência na qualidade do produto final.

\section{CONCLUSÃO}

Os resultados indicaram que a condição sexual influencia as características de qualidade de carne. Animais castrados têm uma melhor qualidade de carne, apresentando carne mais macia e com coloração mais vermelha.

Ciência Rural, v.44, n.5, mai, 2014. 
Tabela 2 - Estimativas dos coeficientes de correlação de Pearson (valores de P) entre as variáveis ${ }^{1}$ endócrinas (Cortisol e ACTH in vivo e post mortem), metabólicas (Glicogênio e Lactato) e as características de qualidade de carne dos machos Nelore castrados e nãocastrados.

\begin{tabular}{|c|c|c|c|c|c|c|}
\hline \multirow[b]{2}{*}{ Variável } & \multicolumn{5}{|c|}{ Animais castrados } & \multirow[b]{2}{*}{ Lactato } \\
\hline & Cortisol_IV & Cortisol_PM & ACTH_IV & ACTH_PM & Glicogênio & \\
\hline $\mathrm{L}^{*} 1 \mathrm{D}$ & $-0,154(0,178)$ & $-0,236(0,020)$ & $-0,490(<0,0001)$ & $-0,102(0,378)$ & $-0,152(0,185)$ & $0,320(0,004)$ \\
\hline a*1D & $0,215(0,059)$ & $0,033(0,772)$ & $0,445(<0,0001)$ & $0,154(0,180)$ & $0,264(0,020)$ & $-0,206(0,071)$ \\
\hline$b^{*} 1 \mathrm{D}$ & $0,223(0,050)$ & $-0,111(0,331)$ & $0,198(0,087)$ & $0,060(0,603)$ & $0,081(0,479)$ & $-0,200(0,079)$ \\
\hline $\mathrm{L} * 7 \mathrm{D}$ & $-0,203(0,075)$ & $-0,189(0,098)$ & $-0,381(0,001)$ & $-0,109(0,345)$ & $-0,042(0,716)$ & $0,307(0,006)$ \\
\hline $\mathrm{a} * 7 \mathrm{D}$ & $0,328(0,003)$ & $-0,292(0,010)$ & $0,349(0,002)$ & $0,174(0,130)$ & $0,192(0,092)$ & $0,241(0,034)$ \\
\hline $\mathrm{b} * 7 \mathrm{D}$ & $-0,018(0,875)$ & $-0,046(0,692)$ & $-0,188(0,103)$ & $0,015(0,897)$ & $0,119(0,299)$ & $-0,082(0,473)$ \\
\hline L*14D & $-0,266(0,023)$ & $-0,323(0,005)$ & $-0,447(<.0001)$ & $-0,140(0,242)$ & $-0,181(0,126)$ & $0,300(0,010)$ \\
\hline$a * 14 D$ & $0,305(0,009)$ & $0,116(0,326)$ & $0,393(0,001)$ & $0,103(0,391)$ & $0,234(0,047)$ & $-0,074(0,534)$ \\
\hline$b * 14 D$ & $-0,191(0,106)$ & $0,094(0,431)$ & $-0,078(0,517)$ & $-0,068(0,571)$ & $0,117(0,322)$ & $-0,092(0,441)$ \\
\hline $\mathrm{pH} 1 \mathrm{H}$ & $0,019(0,868)$ & $0,001(0,990)$ & $-0,248(0,031)$ & $-0,114(0,323)$ & $-0,100(0,381)$ & $-0,156(0,172)$ \\
\hline $\mathrm{pH} 24 \mathrm{H}$ & $-0,207(0,069)$ & $0,205(0,072)$ & $-0,095(0,415)$ & $-0,138(0,231)$ & $-0,209(0,067)$ & $-0,250(0,028)$ \\
\hline PPC1D & $-0,163(0,159)$ & $-0,192(0,097)$ & $-0,334(0,004)$ & $-0,029(0,808)$ & $-0,015(0,897)$ & $0,170(0,142)$ \\
\hline PPC7D & $-0,010(0,930)$ & $0,031(0,798)$ & $-0,138(0,241)$ & $-0,132(0,260)$ & $-0,138(0,235)$ & $-0,041(0,725)$ \\
\hline PPC14D & $-0,095(0,409)$ & $0,012(0,920)$ & $-0,076(0,513)$ & $-0,051(0,661)$ & $-0,055(0,633)$ & $-0,016(0,892)$ \\
\hline FC1D & $-0,170(0,137)$ & $-0,086(0,456)$ & $-0,327(0,004)$ & $-0,101(0,380)$ & $-0,102(0,372)$ & $0,160(0,163)$ \\
\hline FC7D & $-0,162(0,158)$ & $0,177(0,121)$ & $-0,225(0,051)$ & $-0,194(0,090)$ & $-0,241(0,033)$ & $-0,009(0,935)$ \\
\hline FC14D & $-0,083(0,470)$ & $0,094(0,413)$ & $-0,198(0,086)$ & $-0,149(0,196)$ & $-0,264(0,019)$ & $0,081(0,482)$ \\
\hline Variável & Cortisol_IV & Cortisol_PM & ACTH_IV & ACTH_PM & Glicogênio & Lactato \\
\hline $\mathrm{L}^{*} 1 \mathrm{D}$ & $-0,074(0,604)$ & $-0,067(0,643)$ & $-0,1555(0,278)$ & $0,276(0,050)$ & $0,125(0,382)$ & $0,367(0,008)$ \\
\hline $\mathrm{a} * 1 \mathrm{D}$ & $0,195(0,170)$ & $0,286(0,042)$ & $0,287(0,041)$ & $-0,051(0,722)$ & $0,172(0,228)$ & $-0,389(0,005)$ \\
\hline$b * 1 D$ & $0,063(0,659)$ & $0,218(0,124)$ & $0,144(0,313)$ & $0,299(0,033)$ & $0,334(0,017)$ & $-0,201(0,157)$ \\
\hline $\mathrm{L} * 7 \mathrm{D}$ & $-0,076(0,592)$ & $-0,111(0,433)$ & $-0,122(0,389)$ & $0,287(0,039)$ & $0,291(0,037)$ & $0,341(0,013)$ \\
\hline$a * 7 D$ & $0,150(0,289)$ & $0,054(0,706)$ & $0,072(0,612)$ & $-0,228(0,104)$ & $-0,116(0,412)$ & $-0,238(0,090)$ \\
\hline$b^{*} 7 \mathrm{D}$ & $-0,032(0,820)$ & $0,049(0,730)$ & $0,024(0,866)$ & $0,041(0,775)$ & $0,336(0,015)$ & $-0,320(0,021)$ \\
\hline$L^{*} 14 \mathrm{D}$ & $-0,248(0,097)$ & $-0,147(0,331)$ & $-0,156(0,300)$ & $0,291(0,050)$ & $0,196(0,192)$ & $0,488(0,001)$ \\
\hline$a * 14 D$ & $0,342(0,020)$ & $0,234(0,118)$ & $0,259(0,083)$ & $-0,164(0,277)$ & $0,093(0,539)$ & $-0,438(0,002)$ \\
\hline b*14D & $0,005(0,972)$ & $0,137(0,363)$ & $0,280(0,059)$ & $0,100(0,510)$ & $0,439(0,002)$ & $-0,207(0,167)$ \\
\hline $\mathrm{pH} 1 \mathrm{H}$ & $0,604(<0,001)$ & $0,446(0,001)$ & $0,306(0,028)$ & $0,308(0,026)$ & $-0,023(0,873)$ & $-0,234(0,096)$ \\
\hline $\mathrm{pH} 24 \mathrm{H}$ & $-0,171(0,225)$ & $-0,082(0,564)$ & $-0,054(0,702)$ & $-0,064(0,652)$ & $-0,270(0,053)$ & $0,278(0,046)$ \\
\hline PPC1D & $-0,132(0,352)$ & $-0,043(0,761)$ & $-0,052(0,715)$ & $0,130(0,360)$ & $0,234(0,096)$ & $0,033(0,815)$ \\
\hline PPC7D & $-0,054(0,706)$ & $0,057(0,693)$ & $-0,189(0,185)$ & $-0,204(0,152)$ & $0,190(0,182)$ & $-0,149(0,297)$ \\
\hline PPC14D & $0,180(0,203)$ & $0,121(0,391)$ & $0,100(0,482)$ & $0,086(0,546)$ & $-0,040(0,779)$ & $-0,171(0,226)$ \\
\hline FC1D & $-0,142(0,314)$ & $-0,234(0,095)$ & $-0,247(0,077)$ & $-0,186(0,188)$ & $-0,169(0,232)$ & $0,100(0,482)$ \\
\hline FC7D & $-0,131(0,354)$ & $-0,355(0,010)$ & $-0,306(0,027)$ & $-0,287(0,039)$ & $-0,419(0,002)$ & $0,171(0,225)$ \\
\hline FC14D & $0,145(0,305)$ & $-0,277(0,047)$ & $0,020(0,891)$ & $-0,035(0,806)$ & $-0,242(0,085)$ & $0,015(0,914)$ \\
\hline
\end{tabular}

${ }^{1}$ Cortisol $I V$ e ACTH $I V=$ concentração desses hormônios determinadas em amostras de sangue coletadas dos animais no confinamento (in vivo); Cortisol $P M$ e ACTH $P M=$ concentração desses hormônios determinadas em amostras de sangue coletadas no momento da sangria no abate (post mortem); Glicogênio e lactato em $\mu \mathrm{molg}^{-1}$ de tecido; $\mathrm{PPC}=$ perdas por cozimento, em $\%$; $\mathrm{FC}=$ valores de força de cisalhamento em quilogramas.

\section{REFERÊNCIAS}

AMERICAN MEAT SCIENCE ASOCIATION (AMSA). American Meat Science Association and National Live Stock and Meat Board. Chicago, 1995.

COSTA, E.C. et al. Composição física da carcaça, qualidade da carne e conteúdo de colesterol no músculo Longissimus dorsi de novilhos Red Angus superprecoces, terminados em confinamento e abatidos com diferentes pesos. Revista Brasileira de Zootecnia, v.31, p.417-428, 2002. Disponível em: <http://www.scielo.br/pdf/ rbz/v31n1s0/10323.pdf>. Acesso em: 20 jun. 2013. doi: 10.1590/ S1516-35982002000200017.

DIKEMAN, M.E. et al. Longissimus muscle quality, palatabilty and connective tissue histological characteristics of bulls and steers fed different energy levels and slaughtered at four ages. Journal 
of Animal Science, v.63, p.92-101, 1986. Disponível em: <http:// www.journalofanimalscience.org/content/63/1/92.long>. Acesso em: 20 jun. 2013.

FIELD, R.A. Effect of castration on meat quality and quantity. Journal of Animal Science, v.32, p.849-858, 1971. Disponível em: <http://www.ncbi.nlm.nih.gov/pubmed/5571574>. Acesso em: 10 jan. 2013.

GRANDIN, T. Assessment of stress during handling and transport. Journal of Animal Science, v.75, p.249-257, 1997. Disponível em: <http://www.ncbi.nlm.nih.gov/pubmed/9027573>. Acesso em: 20 dez. 2012.

HUFF-LONERGAN, E.; LONERGAN, S.M. Mechanisms of water-holding capacity of meat: the role of postmortem biochemical and structural changes. Meat Science, v.71, p.194-204, 2005. Disponível em: <http://dx.doi.org/10.1016/j. meatsci.2005.04.022>. Acesso em: 20 jun. 2013. doi: 0.1016/j. meatsci.2005.04.022.

JEREMIAH, L.E. et al. The usefulness of muscle color and $\mathrm{pH}$ for segregating beef carcasses into tenderness groups. Meat Science, v.30, p.97-114, 1991. Disponível em: <http://www.ncbi. nlm.nih.gov/pubmed/22061829>. Acesso em: 20 jun. 2013. doi: 10.1016/0309-1740(91)90001-7.

JURUENA, M.F. et al. O eixo hipotálamo-pituitária-adrenal, a função dos receptores de glicocorticóides e sua importância na depressão. Revista Brasileira de Psiquiatria, v.3, p.189201, 2004. Disponível em: <http://dx.doi.org/10.1590/S151644462004000300009>. Acesso em: 11 nov. 2012. doi: 10.1590/ S1516-44462004000300009.

KANITZ, E. et al. A single exposure to social isolation in domestic piglets activates behavioural arousal, neuroendocrine stress hormones, and stress-related gene expression in the brain. Physiology \& Behavior, v.98, p.176-185, 2009. Disponível em: $<$ http://dx.doi.org/10.1016/j.physbeh.2009.05.007>. Acesso em: 15 nov. 2012. doi: 10.1016/j.physbeh .2009.05.007.

KOÉWIN-PODSIADLA, M. et al. Pork quality and methods of its evaluation - a review. Polish Journal of Food and Nutrition Sciences, v.3, p.241-248, 2006. Disponível em: <http://www.cabi. $\mathrm{org} /$ cabdirect/FullTextPDF/2006/20063223514.pdf>. Acesso em: 12 jan. 2013

LAWRIE, R.A. Ciência da carne. Tradução de Jane Maria Rubensan. 6.ed. Porto Alegre: Artmed, 2005. 384p.

$\mathrm{MACH}$, N. et al. Burdizzo prepubertal castration effects on performance, behaviour, carcass characteristics, and meat quality of Holstein bulls fed high-concentrate diets. Meat Science, v.81, p.329-334, 2009. Disponível em: <http://www. journalofanimalscience.org/content/91/3/1129.full>. Acesso em: 20 jun. 2013. doi: $10.2527 /$ jas.2012-5717

MANCINI, R.A.; HUNT, M.C. Current research in meat color. Meat Science, v.71, p.100-121, 2005. Disponível em: $<\mathrm{http}: / /$ dx.doi.org/10.1016/j.meatsci.2005.03.003>. Acesso em: 20 jun. 2013. doi: $0.1016 /$ j.meatsci.2005.03.003.
MORGAN, J.B. et al. Meat tenderness and the calpain proteolytic system in Longissimus muscle of young bulls and steers. Journal of Animal Science, v.71, p.1471-1476, 1993. Disponível em: $<$ http://www.journalofanimalscience.org/content/71/6/1471. long $>$. Acesso em: 07 mar. 2013.

MORMÉDE, P. et al. Breeding for robustness: the role of cortisol. Animal, v.5, p.651-657, 2011. Disponível em: <http://www.ncbi. nlm.nih.gov/pubmed/22439987>. Acesso em: 20 jun. 2013. doi: $10.1017 / \mathrm{S} 1751731110002168$.

MÖSTL, E.; PALME, R. Hormones as indicators of stress. Domestic Animal Endocrinology, v.23, p.67-74, 2002. Disponível em: <http://www.ncbi.nlm.nih.gov/pubmed/12142227>. Acesso em: 17 nov. 2012. doi: S0739-7240(02)00146-7.

PAREDI, G. et al. "Muscle to meat" molecular events and technological transformations: The proteomics insight. Journal of Proteomics, v.75, p.4275-4289, 2012. Disponível em: <http:// dx.doi.org/10.1016/j.jprot.2012.04.011>. Acesso em: 01 jul. 2013. doi: 10.1016/j.jprot.2012.04.01.

PINTO, L.F.B. et al. Qualidade de carne e de carcaça em bovinos da raça Nelore. In: SIMPÓSIO BRASILEIRO DE MELHORAMENTO ANIMAL, 7., 2008, São Carlos, SP. Anais... São Carlos: SBMA, 2008. p.1-4.

RAMOS, E.M; GOMIDE, L.A.M. Avaliação da qualidade de carnes: fundamentos e metodologias. Viçosa-MG: UFV, 2007. 1v.

RESTLE, J. et al. Características das carcaças e da carne de bovinos inteiros ou submetidos a duas formas de castração, em condições de pastagem. Revista da Sociedade Brasileira de Zootecnia, v.25, p.334-344, 1996. Disponível em: <http://www.scielo.br/pdf/ rbz/v37n6/v37n6a16.pdf>. Acesso em: 20 jun. 2013. doi: 10.1590/ S1516-35982008000600016.

RESTLE, J. et al. Características de carcaça de bovinos de corte inteiros ou castrados em diferentes idades. Pesquisa Agropecuária Brasileira, v.29, p.1603-1607, 1994. Disponível em: <http:// seer.sct.embrapa.br/index.php/pab/article/viewFile/4213/1504>. Acesso em: 20 jun. 2013.

RODRIGUES, V.C.; ANDRADE, I.F. Características físicoquímicas de bubalinos e de bovinos castrados e não-castrados. Revista Brasileira de Zootecnia, v.33, p.1839-1849, 2004. Disponível em: <http://dx.doi.org/10.1590/S151635982004000700023>. Acesso em: 05 nov. 2012. doi: 10.1590/ S1516-35982004000700023.

VAZ, F.N.; RESTLE, J. Aspectos qualitativos da carcaça e da carne de machos Hereford, inteiros ou castrados, abatidos aos quatorze meses. Revista Brasileira de Zootecnia, v.29, p.1894-1901, 2000. Disponível em: <http://www.scielo.br/pdf/rbz/v29n6/5723.pdf>. Acesso em: 01 dez. 2012.

WULF, D.M. et al. Relationships among glycolytic potencial, dark cutting (dark, firm, and dry) beef, and cooked beef palatability. Journal of Animal Science, v.80, p.1895-1903, 2011. Disponível em: <http://www.ncbi.nlm.nih.gov/pubmed/12162657>. Acesso em: 01 dez. 2012. 